\title{
ALERO LAS MORRENAS 1: EVIDENCIAS DE CULTÍGENOS ENTRE CAZADORES RECOLECTORES DE FINALES DEL PERÍODO ARCAICO EN CHILE CENTRAL
}

\author{
LAS MORRENAS 1 ROCKSHELTER: EVIDENCE FOR CULTIGENS \\ AMONG HUNTER GATHERERS OF THE LATE ARCHAIC \\ PERIOD IN CENTRAL CHILE
}

\author{
María Teresa Planella O. , Luis E. Cornejo B. ${ }^{* *}$ y Blanca Tagle A. ${ }^{* * *}$
}

\begin{abstract}
Los resultados de estudios arqueobotánicos realizados en el sitio Las Morrenas 1, localizado en la cuenca andina del río Maipo en Chile Central, demuestran la presencia de restos carbonizados de quínoa (Chenopodium quinoa Willd.) en un contexto de cazadores recolectores del momento más tardío del período Arcaico, con fechas de 3.250 a 2.980 años cal. a.p. Estas evidencias están presentes en toda la secuencia de ocupación del sitio, que incluye depósitos del período Alfarero Temprano en los niveles superiores. Los materiales indican que en ambos períodos se utilizó el asentamiento como campamento de tareas específicas de carácter estacional. Se discute el contexto regional y areal para la asociación entre cultígenos y poblaciones de finales del período Arcaico.

Palabras claves: estudios arqueobotánicos, Chenopodium quinoa, Zea mays, período Arcaico, cordillera de Chile Central.
\end{abstract}

Archaeobotanical studies from Las Morrenas 1 site, located in the Andean basin of the Maipo river in Central Chile, demonstrated the presence of charred remains of quinoa (Chenopodium quinoa Willd.) in a context of hunter gatherers from the end of the Archaic Period, with dates that ranged from 3250 to 2980 cal. years B.P. This evidence continued to be found through the occupation sequence observed at the site, which included Early Ceramic Period deposits at the upper levels. Archaeological materials indicated that in both periods the settlement was used for specific activities of seasonal character. The regional and local contexts of associations between cultigens and populations at the end of the Archaic Period are discussed.

Key words: archaeobotanical studies, Chenopodium quinoa, Archaic Period, mountain range, Central Chile.

En el transcurso de investigaciones arqueológicas desarrolladas en la cordillera andina de Chile Central se ha descubierto y estudiado una variedad de asentamientos que representan una larga secuencia de ocupación humana prehistórica. Esta se extiende por más de 11.000 años, llegando hasta el inicio de la colonización española de esta parte del Mundo (Cornejo y Simonetti 1992, 1993, 1997; Cornejo et al. 1998; Cornejo y Sanhueza 2003).

Dentro de esta secuencia, uno de los momentos mejor representados corresponde al período que va entre los 3.000 a 400 años a.C. o Arcaico IV (Cornejo et al. 1998), el cual ha sido estudiado en una serie de aleros rocosos emplazados entre los 1.000 y $2.600 \mathrm{~m}$ de altitud en valles y laderas de los principales afluentes del río Maipo. Estos sitios arqueológicos representan a diversos tipos de campamentos (bases, de tareas específicas y ocasionales) que formaron parte de circuitos de movilidad que incluían tanto la cordillera como, probablemente, regiones vecinas (Cornejo et al. 1998; 2000a).

La interpretación del contexto arqueológico ha permitido describir para dicho momento un modo de vida basado en la economía de caza y recolección y una gran movilidad regional (Cornejo et al. 1998; 2000b), que puede ser clasificada principalmente como logística (Binford 1980). Esta caracterización es altamente coherente con la concepción tradicional que hasta hace poco tiempo se tenía de las sociedades de cazadores recolectores prehortícolas en general y, particularmente, para Chile Central. Sin embargo, uno de los últimos sitios estudiados en la región cordillerana, el alero Las Morrenas 1, entregó evidencias que indican la pre-

\footnotetext{
* Sociedad Chilena de Arqueología. El Amancai 505, Las Condes. mtplanella@123mail.cl.

** Museo Chileno de Arte Precolombino. Bandera 361, Santiago de Chile. lcornejo@ museoprecolombino.cl.

*** Sociedad Chilena de Arqueología. Casilla 93, San Fernando. mbtagle@ yahoo.es
} 
sencia de Chenopodium quinoa, entre los recursos comestibles recuperados en los niveles más antiguos de este asentamiento, que de acuerdo a las características del contexto y las fechas arqueométricas obtenidas, debieran corresponder a cazadores recolectores arcaicos (Galarce y Peralta 2003; Planella y Tagle 2004; Tagle y Planella 2002). El objetivo del presente artículo es caracterizar estos hallazgos y discutir sus implicancias para el entendimiento de los modos de vida de las poblaciones que se encuentran cercanas al vértice entre la economía pre y posthortícola.

\section{El Sitio Las Morrenas 1}

Este asentamiento está constituido por un pequeño alero de unos $8,75 \mathrm{~m}^{2}$ de superficie cubierta y el cono de basuras que se formó alrededor de su entrada. Se aloja en un bloque errático andesítico que descansa sobre un antiguo sistema de morrenas que caracterizan el curso medio del río Yeso (UTM 627341 N 396500 E), tributario del río Maipo (Figura 1). El sitio se emplaza a los $2.450 \mathrm{~m}$ de altitud en un ambiente altoandino que en esta localidad presenta una serie de lagunas de distinto ta-

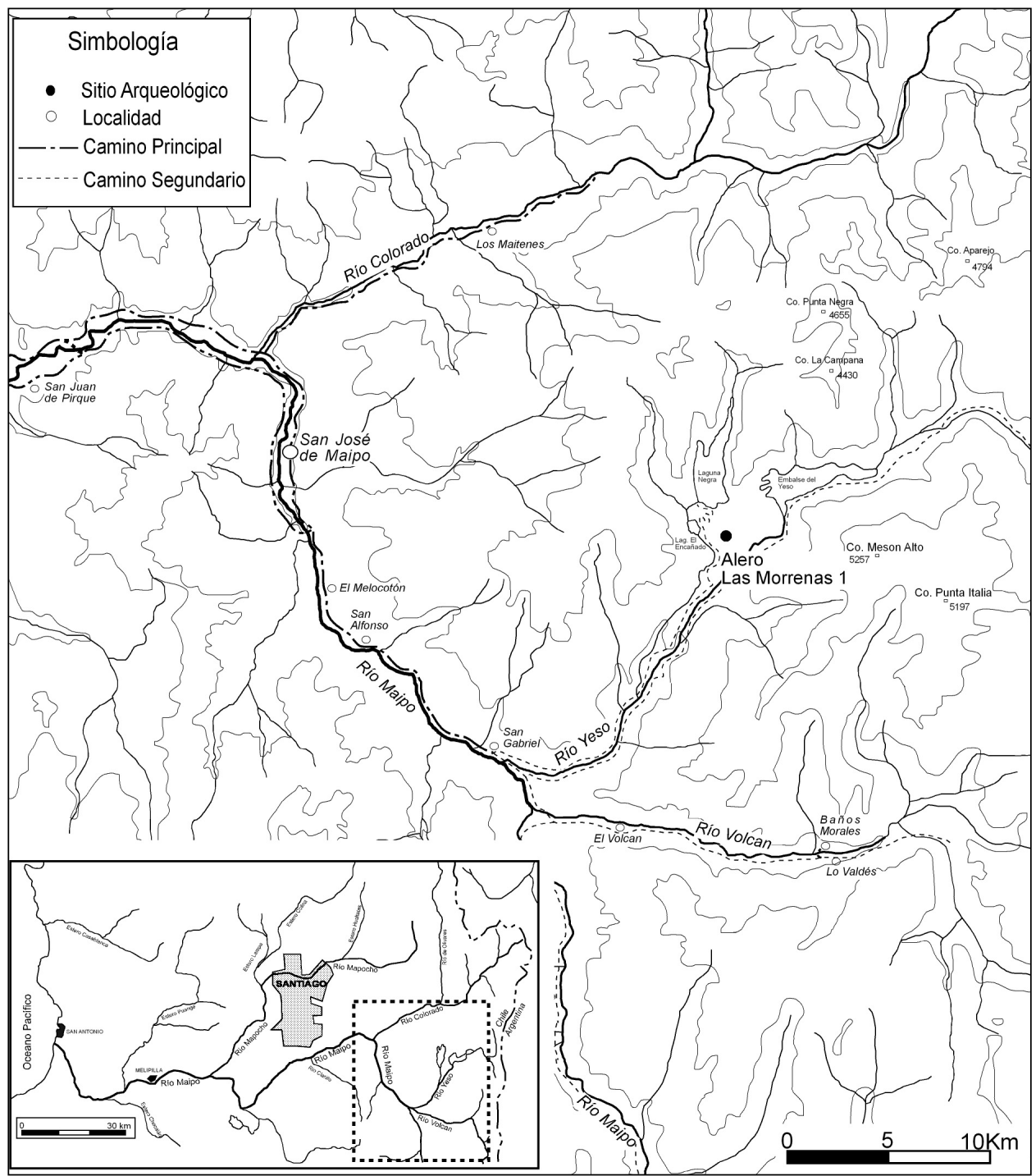

Figura 1. Cuenca cordillerana del río Maipo y localidad de Las Morrenas. Location of the Rio Maipo basin and Las Morrenas archaeological site. 
maño, una de las cuales se encontraba a los pies del sitio en el momento de verificarse las primeras ocupaciones y que hoy está completamente seca.

La vegetación del área del yacimiento comparte condiciones y características tanto de la franja ecológica del "piso andino" (2.700 a $3.100 \mathrm{msm}$ ) con dominio de plantas leñosas, en cojines y longevas, como del "piso subandino" (2.100 a 2.700 $\mathrm{msm}$ ) con una alta diversidad de especies, varias de las cuales son hierbas anuales; entre éstas, la familia Poaceae es la que presenta mayor cobertura en dicho ecosistema. Las asociaciones vegetacionales que corresponden al "piso andino" son: Anarthrophyllum gayanum-Nassauvia heterophylla, la asociación Anarthrophyllum gayanum-Laretia acaulis, y la asociación Laretia acaulis-Anarthrophyllum cumingii. Por su parte al "piso subandino" corresponderían las asociaciones $\mathrm{Chu}$ quiraga oppositifolia-Nassauvia axillaris-Ephedra andina, Nassauvia axillaris-Chuquiraga oppositi- folia y Chuquiraga oppositifolia-Tetraglochin alatum (Hoffman et al. 1998), que corresponde aproximadamente a la "estepa altoandina de la cordillera de Santiago" de Gajardo (1994). A su vez los bordes de lagunas proveen de abundante flora de tipo palustre.

Las excavaciones arqueológicas en el sitio se concentraron principalmente en la parte exterior del alero ya que el sector cubierto, pese a tener una superficie utilizable, presenta en general una altura muy baja -en muchas partes menor a $50 \mathrm{~cm}$-que dificulta la realización de tareas de excavación. Sin embargo en el exterior del sitio se aprecia un montículo de sedimentos y desechos de la ocupación, que con un diámetro de cerca de $8 \mathrm{~m}$ y un espesor de 80 $\mathrm{cm}$ se presentaba favorable para una intervención estratigráfica. Sobre este montículo exterior y parcialmente en la boca del alero se trazaron inicialmente dos cuadrículas de $1 \times 1,5 \mathrm{~m}$, que posteriormente se ampliaron en 1 m (Figura 2). La excavación

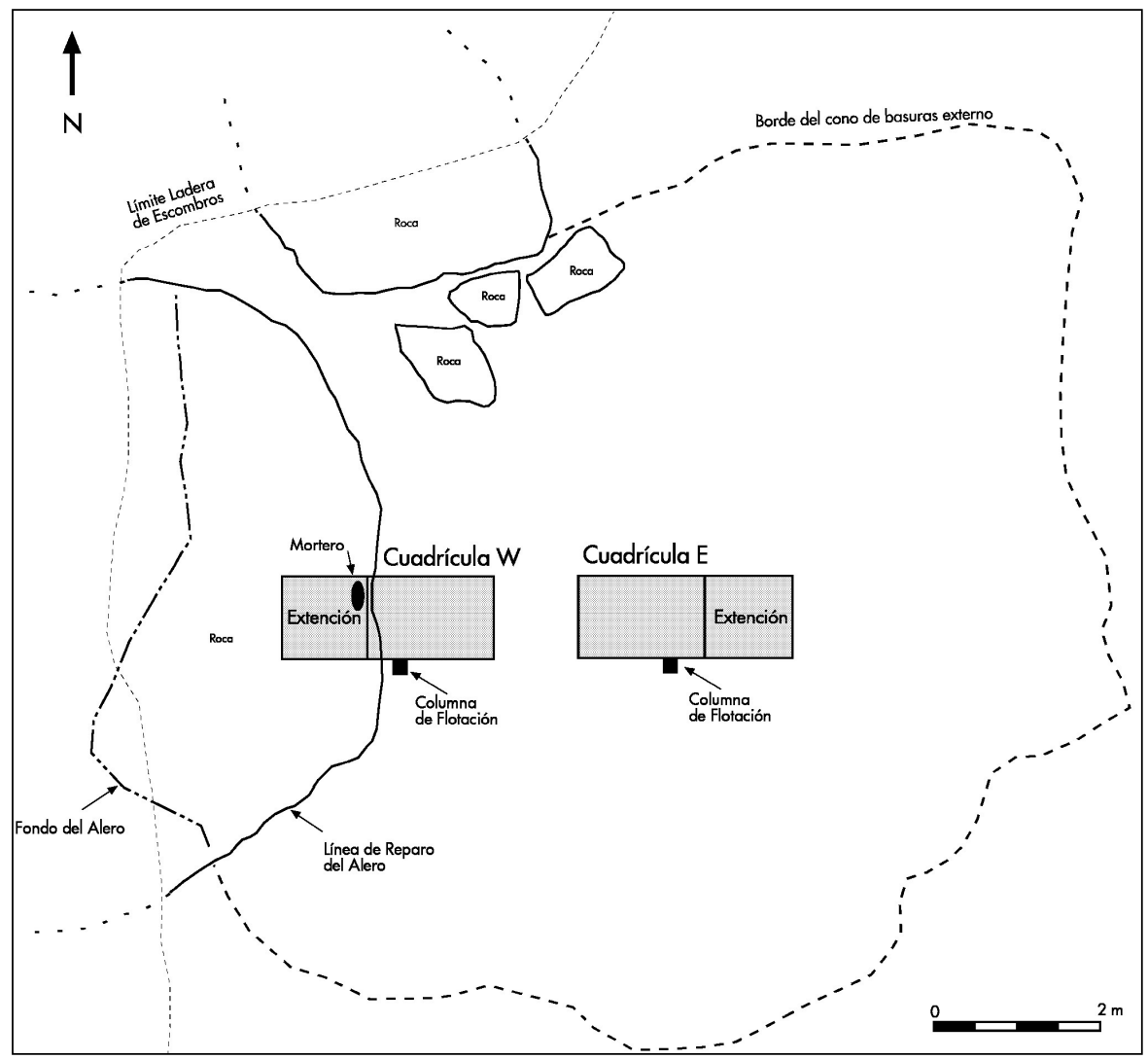

Figura 2. Plano de las excavaciones y ubicación de las columnas de muestreo para flotación en el alero Las Morrenas 1.

Map of the excavated areas and flotation sampling locations at Las Morrenas 1 rockshelter. 
se realizó siguiendo la estratigrafía natural y segregando niveles artificiales de $10 \mathrm{~cm}$ dentro de cada estrato. Con esto se obtuvo una muestra de $5 \mathrm{~m}^{2}$, lo que corresponde a un 8,5 \% de la superficie de mayor concentración de materiales (cono de desechos e interior del alero).

Todos los depósitos fueron harneados en una malla de $3 \mathrm{~mm}$ de calado y, posteriormente, un $25 \%$ de los sedimentos ya tratados fueron nuevamente harneados en una malla de $1 \mathrm{~mm}$ de calado. Esto permitió recolectar una muestra significativa de los desechos culturales desde un tamaño superior a $1 \mathrm{~mm}$.

A partir de los depósitos excavados se pudo segregar dos ocupaciones humanas de distintos momentos. En los niveles superiores, hasta $60 \mathrm{~cm}$ de profundidad, se verificó la presencia de restos que, si bien no se han datado arqueométricamente hasta ahora, desde el punto de vista contextual serían sincrónicos al llamado período Alfarero Temprano (en adelante PAT) que caracteriza la prehistoria de Chile Central entre los años 2.300 y 1.100 a.p. Esta asignación cronológica se basa principalmente en la tipología de los abundantes fragmentos cerámicos que caracterizan estos niveles. Por su parte, en los estratos inferiores del asentamiento, entre 70 y $100 \mathrm{~cm}$, se detectó la presencia de una ocupación más antigua, la cual fue fechada en 3.400-3.210 cal. a.p. (BETA 127529) y 3.675-3.450 años cal. a.p. (BETA 127528), ambas con una probabilidad de 95,4 \% (Galarce y Peralta 2003).

\section{El estudio arqueobotánico de Las Morrenas 1}

Debido a que esta intervención arqueológica del sitio se encontraba dentro de un plan de investigación interdisciplinaria con arqueobotánica, se procedió a implementar una estrategia adecuada a las características del sitio, las condiciones biogeográficas del entorno y al potencial de depósitos que quedó al descubierto durante las excavaciones.

Se extrajo dos columnas de $20 \times 20 \mathrm{~cm}$ con muestras de tierra para flotación cada $10 \mathrm{~cm}$ hasta el nivel estéril de limo blanco amarillento (100 y $110 \mathrm{~cm}$ de profundidad), las que se practicaron en el perfil sur de las cuadrículas Oeste y Este, por presentar allí menos interrupción de piedras en la secuencia del muestreo. Conjuntamente, se recuperó de modo íntegro los sedimentos de un rasgo de fogón de contorno irregular, de 70-80 cm de diámetro y localizado a $44-52 \mathrm{~cm}$ de profundidad en la cuadrícula Este; y de la boca del alero se obtuvo una muestra de tierras asociadas al nivel de apoyo de la base de un molino de piedra, a 55-60 $\mathrm{cm}$; y otra a $120 \mathrm{~cm}$ en un sector con presencia de tres manos de moler, una de ellas cubierta con pigmento rojo.

En total se sometieron a flotación en una máquina especialmente diseñada para ello 87,75 litros: 40,5 litros de la cuadrícula Este y 47,25 litros de la Oeste. En laboratorio, las fracciones liviana y pesada de la muestra flotada fueron analizadas bajo lupa binocular Nikon SMZ-1B y para la identificación se efectuó una comparación con muestras de referencia carbonizadas y semillas y frutos de las especies recolectadas en el área de sitio. El estudio se realizó en macrorrestos carbonizados, que es la condición en que se encuentran los vestigios botánicos arqueológicos en la zona central, y algunas especies no carbonizadas introducidas por distintos agentes en las tierras analizadas y que ayudan a reconocer flora actual y grados de contaminación de las muestras ${ }^{1}$.

El estado de conservación se considera muy bueno, no sólo en relación a frutos y semillas con ciertas características de dureza, sino también en aquellos de mayor fragilidad como lo son Chenopodiaceae, Poaceae, Berberidaceae y especies de Nicotiana. De los restos, sólo mostraron mayor fragmentación y deterioro cariopsis de Zea mays L. recuperados de los niveles cerámicos, y de $\mathrm{Cu}$ curbitaceae.

La fracción liviana, el material orgánico menor de $2 \mathrm{~mm}$, concentró la mayor cantidad de las semillas y frutos recuperados, incluyendo los cultígenos, junto a fragmentos mínimos de maderas, restos óseos y desechos líticos muy pequeños. Especímenes de la flora actual, raicillas y fecas de roedores fueron también registrados en esta fracción.

La fracción pesada, principalmente con elementos mayores a $2 \mathrm{~mm}$, además del registro botánico, sirvió para complementar la información cultural obtenida en las excavaciones arqueológicas. Asimismo, para el Arcaico IV, lascas y desechos de talla que confirman las materias primas y la intensidad del trabajo en lítico; trozos ligeramente mayores de maderas carbonizadas y en algunos casos con huellas de cortes; fragmentos óseos de fauna menor del área del asentamiento, y de $\mathrm{Ca}$ melidae como producto efectivo de la actividad de cazadores cordilleranos. En el PAT, se rescató elementos diagnósticos como fragmentos cerámicos 
y una cuenta de collar de malaquita; además de elementos líticos indicadores de continuidad en los patrones de tallado en distintas materias primas, y depositaciones calcáreas y tierra quemada en contextos de fogón. Como aporte complementario para entender el sustrato natural del asentamiento, la fracción pesada permitió visualizar los elementos constituyentes que confirman y caracterizan la matriz del suelo.

Con el propósito de disponer de una muestra de referencia de la vegetación que actualmente se desarrolla en dicho ecosistema altoandino, se pro- cedió a realizar, durante las excavaciones en temporada de verano (enero de 1999), una colecta de muestras de especies de la flora asociada espacialmente al sitio, en dos transectas de aproximadamente $600 \mathrm{~m}$ de diámetro, encontrándose muchas de ellas con sus frutos maduros (Tabla 1).

\section{Resultados}

La información estratigráfica y de adscripción cultural que aportó la excavación de las cuadrícu-

Tabla 1. Plantas colectadas en un diámetro de $600 \mathrm{~m}$ en el entorno del sitio Las Morrenas 1.

Plants collected from a $600 \mathrm{~m}$ area surrounding the Las Morrenas 1 rockshelter site.

\begin{tabular}{|c|c|c|c|}
\hline Familia & Taxón & Nombres vulgares & Usos \\
\hline Apiaceae & Laretia acaulis & Llareta & Combustión \\
\hline Asclepiadaceae & $\begin{array}{l}\text { Cynanchium nummularifolium } \\
\text { Baccharis sp. }\end{array}$ & Pahueldum & \\
\hline Asteraceae & $\begin{array}{l}\text { Chuquiraga oppositifolia } \\
\text { Haplopappus schumannii } \\
\text { Mutisia sinuata } \\
\text { Solidago chilensis } \\
\text { Senecio } \text { sp. }\end{array}$ & $\begin{array}{l}\text { Yerba blanca } \\
\text { Clavel del campo, granadilla } \\
\text { Huellen, filil, filel }\end{array}$ & \\
\hline Berberidaceae & $\begin{array}{l}\text { Berberis empetrifolia } \\
\text { Berberis montana }\end{array}$ & $\begin{array}{l}\text { Uva de cordillera, palo amarillo, } \\
\text { monte negro } \\
\text { Palo amarillo }\end{array}$ & $\begin{array}{l}\text { Fruto comestible, maduro } \\
\text { entre diciembre y abril. } \\
\text { Fruto comestible, maduro } \\
\text { entre diciembre y abril. }\end{array}$ \\
\hline Boraginaceae & Cryptantha capituliflora & Papua & \\
\hline Cyperaceae & Eleocharis palustris & Eleocharis del pantano & $\begin{array}{l}\text { Los rizomas de algunas } \\
\text { especies son comestibles }\end{array}$ \\
\hline Ephedraceae & Ephedra andina & $\begin{array}{l}\text { Pingo-pingo, solupe trasmontana, } \\
\text { sulupe }\end{array}$ & $\begin{array}{l}\text { Frutos comestibles, } \\
\text { maduros en temporada } \\
\text { de verano. }\end{array}$ \\
\hline Iridaceae & Olsynium sp. & Ñuño, quilmo, huilmo & \\
\hline Lamiaceae & Stachys sp. & $\begin{array}{l}\text { Hierba santa } \\
\text { Lahuen-lahuen }\end{array}$ & Medicinal \\
\hline Papilionaceae & Adesmia sp. & Jarilla, espuma & \\
\hline Plumbaginaceae & Armeria maritima & & \\
\hline Rosaceae & Acaena sp. & & \\
\hline Rubiaceae & Galium eriocarpus & Lengua de gato & \\
\hline Scrophulariaceae & Euphrasia andicola & Eufrasia amarilla & \\
\hline
\end{tabular}

Nota: Se obtuvieron, además, algunos especímenes de Juncaceae al borde de una laguna y cuatro especies de Poaceae con espiguillas maduras. 
las constituyó un favorable referente para analizar los restos vegetales dejados por las poblaciones tanto del Arcaico IV como del PAT y con ello intentar reconocer indicadores sustantivos, desde la arqueobotánica, para apoyar la caracterización cultural de ambos momentos.

En la Tabla 2 se muestran los resultados del análisis efectuado en los restos vegetales contenidos en la columna de muestreo practicada en la cuadrícula Este. En primer lugar, se aprecian depósitos muy consistentes entre los 10 y $60 \mathrm{~cm}$ de profundidad, que se adscriben a ocupaciones del PAT; y otras entre los 70 y $100 \mathrm{~cm}$ que corresponden al Arcaico IV. Entre ambos momentos cronológico-culturales, la muestra correspondiente al nivel $60-70 \mathrm{~cm}$ presenta escasos restos botánicos en la fracción fina, lo que podría estar asociado al cambio cultural que se produce en la interfase entre el Arcaico IV y el PAT. No obstante, en la frac- ción gruesa de la flotación y en los depósitos excavados no se aprecian cambios sustantivos en la cantidad de materiales arqueológicos.

Desde el nivel inferior de esta cuadrícula, en contexto arcaico, se constata la presencia de vestigios carbonizados de Chenopodium quinoa ${ }^{2}$ de tamaño pequeño, entre 1 y 1,4 mm (Figura 3) asociados a vegetación silvestre, principalmente Poaceae. Si se considera la densidad que representan los 12 ejemplares obtenidos del nivel más bajo de la columna, incluidos en sólo un litro de tierra, ya que la presencia de grandes piedras en la base del yacimiento impidió una muestra mayor, y que con respecto a las demás especies del mismo nivel representan un $11,88 \%$, es posible afirmar que este hallazgo es de gran relevancia en la discusión sobre la presencia de cultígenos desde algún momento del Arcaico IV. En los depósitos de procedencia vegetal de este período, graficados en las columnas entre

Tabla 2. Macrorrestos en columna de muestreo Cuadrícula Este Macro remains from sample column of East quadrant.

\begin{tabular}{|c|c|c|c|c|c|c|c|c|c|c|}
\hline Nivel & $0-20$ & $20-30$ & $30-40$ & $40-50$ & $50-60$ & $60-70$ & $70-80$ & $80-90$ & $90-100$ & Total \\
\hline Litros & 2 & 4 & 5 & 3 & 4,5 & 4 & 4 & 2,5 & 1 & 30 \\
\hline Amaranthaceae & & & & & & & & & $1^{\wedge}$ & 1 \\
\hline Armeria sp. & & & $1 *$ & & & & & & & 1 \\
\hline Asteraceae & & & $2^{*}$ & & & & $6^{*}$ & & & 8 \\
\hline Berberis sp. & & & $8^{*}$ & $1^{*}$ & & & & & & 9 \\
\hline Bromus sp. & & $2 *$ & $9^{*}$ & $6^{*}$ & & & $3 *$ & & $8^{*}$ & 28 \\
\hline Chenopodium album & $20^{\wedge}$ & $184^{\wedge}$ & $59^{\wedge}$ & & & & & $1^{\wedge}$ & $1^{\wedge}$ & 265 \\
\hline Chenopodium quinoa & $4 *$ & $7 *$ & $21^{*}$ & $19 *$ & $9^{*}$ & & $2 *$ & $4^{*}$ & $12^{*}$ & 78 \\
\hline Chenopodium sp. & $23^{\wedge}$ & $22^{\wedge}$ & $50^{\wedge}$ & $2^{*}$ & $7^{\wedge}$ & & $1^{\wedge}$ & $1 *$ & & 106 \\
\hline Cucurbitaceae & & $2 *$ & & $6^{*}$ & $6^{*}$ & & $4^{*}$ & $1 *$ & & 19 \\
\hline Cyperaceae & & $3 *$ & $4^{*}$ & & & & & & $1^{*}$ & 8 \\
\hline Discaria chacaye & $4^{*}$ & $1 *$ & & $2^{*}$ & $2 *$ & $1 *$ & $7 *$ & $3 *$ & $2^{*}$ & 22 \\
\hline Guindilla trinervis & $1^{*}$ & & $26^{*}$ & & $11^{*}$ & & & $1^{*}$ & & 39 \\
\hline Juncaceae & & $1^{*}$ & & & & $1^{*}$ & & & & 3 \\
\hline Nicotiana sp. & & $16^{\wedge} 3^{*}$ & $1^{\wedge} 3^{*}$ & $1^{\wedge} 1^{*}$ & $1^{\wedge} 1^{*}$ & & & & & 27 \\
\hline Olsynium sp. & & & $2 *$ & & & & & & & 2 \\
\hline Papilionaceae & & & $17^{*}$ & $3 *$ & $2 *$ & & $3^{*}$ & & & 25 \\
\hline Poaceae(ni) & $4 *$ & $15^{*}$ & $30^{*}$ & $42^{*}$ & $36^{*}$ & $6^{*}$ & $33^{*}$ & $1^{\wedge} 36^{*}$ & $17^{*}$ & 220 \\
\hline Scirpus sp. & & $1 *$ & $1^{*}$ & $2^{*}$ & $1 *$ & $1^{*}$ & $1^{*}$ & & $1^{*}$ & 8 \\
\hline Zea mays & & $3 *$ & & $5^{*}$ & $1 *(?)$ & & & & & 9 \\
\hline No identificados & $27 *$ & $64 *$ & $45^{*}$ & $25^{*}$ & $33^{*}$ & & $43^{*}$ & $35^{*}$ & $58^{*}$ & 330 \\
\hline Total & 83 & 325 & 279 & 115 & 110 & 9 & 103 & 83 & 101 & 1208 \\
\hline
\end{tabular}

$*$ = carbonizados

$\wedge=$ no carbonizados

(ni) $=$ no identificada 


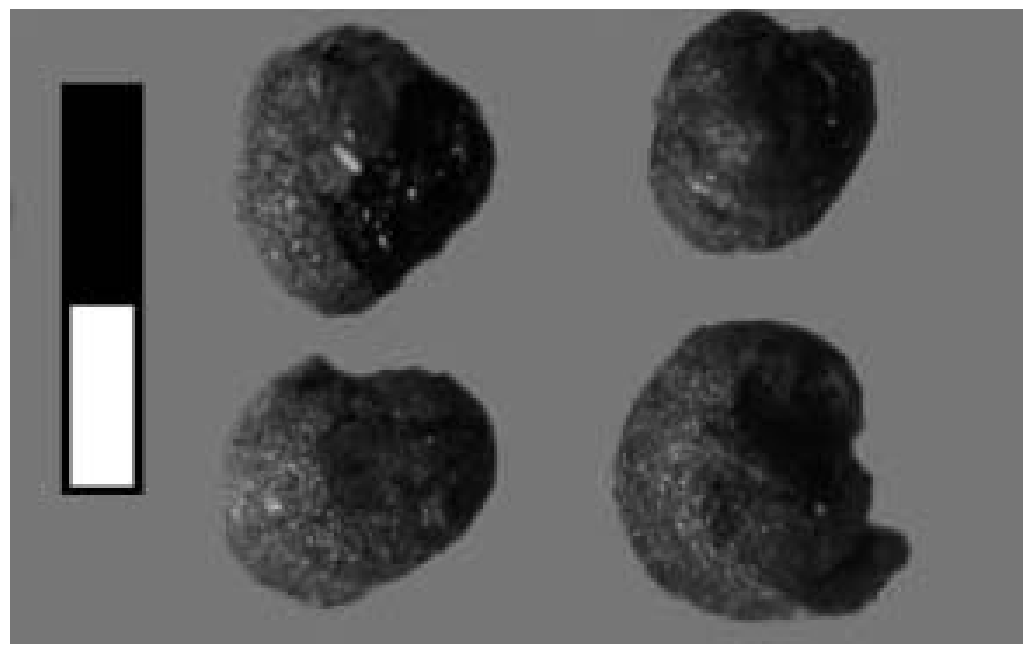

Figura 3. Especímenes carbonizados de Chenopodium quinoa de los niveles arcaicos (8x) (escala 4mm).

Charred specimens of Chenopodium quinoa from archaic levels (8x) at Las Morrenas 1 rockshelter (scale 4mm).

los 70 y $100 \mathrm{~cm}$ de profundidad en la cuadrícula Este y entre 60 y $110 \mathrm{~cm}$ en la Oeste, Chenopodium quinoa alcanza a $6,27 \%$ y $8,69 \%$ respectivamente, incidencia solamente superada por Poaceae. A esto se debe agregar que en la columna de muestreo efectuada en la cuadrícula contigua o cuadrícula Oeste (Tabla 3) el nivel más profundo corresponde a 100$110 \mathrm{~cm}$; al igual que en la Este y, por la misma razón ya mencionada, sólo se logró obtener una muestra de un litro. Esta muestra también proporcionó 12 ejemplares carbonizados del mencionado cultígeno, con tamaños de entre 1,0 a $1,2 \mathrm{~mm}$ y representando un $14,81 \%$ del total de 81 macrorrestos vegetales recuperados en dicho nivel.

Se puede apreciar en ambas tablas que a diferencia de las ocupaciones del PAT, en los niveles arcaicos no aparece representado Zea mays, tampoco Nicotiana sp., Berberis sp. ni la variedad en especies de la cubierta vegetacional altoandina que se registran en los niveles cerámicos. A su vez, Scirpus sp. se manifiesta desde los niveles de base de la ocupación, lo cual estaría acorde por la proximidad del sitio a sectores lacustres.

Como producto de la flotación, de entre los depósitos arcaicos y a partir de los niveles más antiguos, se recuperó además osteofauna (Rhodentiae y Camelidae), incluso fecas de roedores de distintas especies en gran cantidad, y material lítico de desecho, producto de la talla principalmente de cuarzo, obsidiana y cristal de roca. Los residuos de maderas carbonizadas son moderadamente abundantes y provienen de ramas delgadas de especies arbustivas, algunas de las cuales presentan marcas nítidas efectuadas con algún tipo de instrumento cortante. Entre estas especies leñosas, el fruto consistente en una cápsula tricoca elipsoidal de Discaria chacaye, arbusto nativo de las Rhamnaceae y que proporciona una excelente y prolongada combustión, aparece carbonizado desde los primeros niveles del depósito (Figura 4). Este arbusto ha sufrido una explotación subactual indiscriminada, por lo que se encuentran escasos ejemplares en el área estudiada.

En relación a cómo se manifiestan los depósitos de restos vegetales en el PAT, las columnas correspondientes a los niveles con alfarería, graficadas entre los 10 y $60 \mathrm{~cm}$ de profundidad en las Tablas 2 y 3 , señalan cambios en comparación con los del período Arcaico. Se visualiza una mayor variedad en la flora representada, tanto de la cubierta vegetal como del matorral del ecosistema altoandino. Es posible argumentar que las condiciones ambientales en este período habrían presentado mayor humedad, siendo más favorables y similares a las actuales para permitir un desarrollo de vegetación más variado (Heusser 1983), y que esta mayor disponibilidad de recursos a la vez habría ampliado el espectro de las alternativas y decisiones tomadas por los grupos del PAT con respecto al aprovechamiento de su entorno. 
Tabla 3. Macrorrestos en columna de muestreo Cuadrícula Oeste.

Macro remains from sample column of West Quadrant.

\begin{tabular}{|c|c|c|c|c|c|c|c|c|c|c|}
\hline \multirow[b]{2}{*}{ Nivel } & \multirow[b]{2}{*}{$10-20$} & \multicolumn{4}{|c|}{ Período Alfarero Temprano } & \multicolumn{5}{|c|}{ Período Arcaico IV } \\
\hline & & $20-30$ & $30-40$ & $40-50$ & $50-60$ & $60-70$ & $70-80$ & $80-100$ & $100-110$ & Total \\
\hline Litros & 4 & 5 & 5 & 4 & 4 & 5 & 5 & 3 & 1 & 36 \\
\hline Amaranthaceae & $2^{\wedge}$ & & $1 *$ & & & & & & & 3 \\
\hline Armeria sp. & & $1 *$ & & & & & & & & 1 \\
\hline Asteraceae & $1^{*}$ & & $2 *$ & $2^{*}$ & $3^{*}$ & $3^{*}$ & $3^{*}$ & $2 *$ & $2^{*}$ & 18 \\
\hline Berberis sp. & & & & & & & & & & \\
\hline Bromus sp. & $1^{*}$ & $3^{*}$ & $3 *$ & & & $17 *$ & & & & 24 \\
\hline Chenopodium sp. & $3^{\wedge}$ & $9^{*} 6^{\wedge}$ & $3^{\wedge}$ & $5^{*} 2^{\wedge}$ & & $1^{*}$ & & $3 *$ & $1^{*}$ & 33 \\
\hline Ch. quinoa & $2 *$ & & $9 *$ & $3^{*}$ & $4 *$ & $5^{*}$ & $4^{*}$ & $5^{*}$ & $12^{*}$ & 44 \\
\hline Ch. album & $6^{\wedge}$ & $19^{\wedge}$ & & & & & & & & 25 \\
\hline Cucurbitaceae & $1^{*}$ & $2 *$ & & & $3^{*}(?)$ & $1^{*}$ & & $3 *(?)$ & $2 *(?)$ & 12 \\
\hline Discaria chacaye & $2 *$ & & $4^{*}$ & $1 *$ & & $1 *$ & $2^{*}$ & $1 *$ & & 11 \\
\hline Cyperaceae & & $1^{*}$ & $1^{*}$ & & $4^{*}$ & $4^{*}$ & $1^{*}$ & & $1^{*}$ & 12 \\
\hline Guindilia trinervis & & $2^{*}$ & & & & & & $4 *$ & & 6 \\
\hline Juncaceae & $1^{*}$ & & & & & & & & $3^{*}$ & 4 \\
\hline Nicotiana sp. & $2^{*} 3^{\wedge}$ & $2^{*} 6^{\wedge}$ & & $2^{*}$ & $4 *$ & $2 *$ & $3^{*}$ & & & 24 \\
\hline Olsynium sp. & & & & & & & & & & \\
\hline Oxalis sp. & & & & & & & 1 desec. & $1^{\wedge}$ & & 2 \\
\hline Papilionaceae & & $1^{*}$ & $2 *$ & $2^{*}$ & $1 *$ & & & $2^{*}$ & & 8 \\
\hline Poaceae (ni) & $5^{*}$ & $27 *$ & $26^{*}$ & $31^{*}$ & & $13 *$ & $34^{*}$ & $55^{*} 1^{\wedge}$ & $34^{*} 1^{\wedge}$ & 227 \\
\hline Scirpus sp. & $2 *$ & $1 *$ & & $5^{*}$ & $1^{*}$ & $1^{*}$ & & & & 10 \\
\hline Solanaceae & & & & $2^{*}$ & & & & & & 2 \\
\hline Stachys sp. & $3 *$ & $5^{*}$ & $3 *$ & $1^{*}$ & & & & & & 12 \\
\hline Zea mays & & $3 *(?)$ & $3 *(?)$ & & & & & & & 6 \\
\hline No identificados & $36^{*}$ & $48^{*}$ & $27^{*}$ & $24^{*}$ & $27^{*}$ & $7 *$ & $16^{*}$ & $22 *$ & $25^{*}$ & 232 \\
\hline Total & 70 & 136 & 84 & 80 & 47 & 55 & 64 & 99 & 81 & 716 \\
\hline
\end{tabular}

$*$ = carbonizados

$\wedge$ = no carbonizados

$($ ni) $=$ no identificada
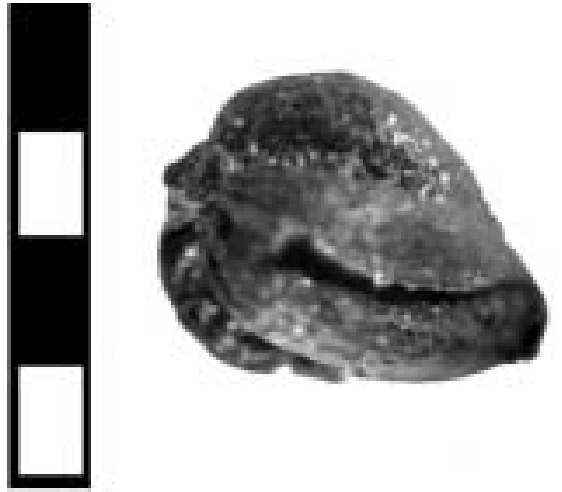

Figura 4. Espécimen carbonizado de Discaria chacaye (8x). Detalle del fruto consistente en una cápsula tricoca elipsoidal (escala 2mm).

Charred specimen from Discaria chacaye (8x). Details of the fruit characterized by ellipsoidal capsule tricoca (scale $2 \mathrm{~mm}$ ).
En este período, pese a estar integrando niveles con menor profundidad, la mayoría de los macrorrestos recuperados están carbonizados, es decir, de algún modo llegaron a integrar los contextos culturales del asentamiento, ya sea por la acción antrópica o por medio de agentes naturales de depositación, permaneciendo distinguibles hasta la actualidad (Evans y O'Connor 2001). Vemos por otra parte que varias de las especies nativas mencionadas en el ejercicio de recolección de muestras de referencia de vegetación actual, aparecen carbonizadas en estos contextos, incluso algunas desde el Arcaico IV, lo que aporta una información relevante acerca del paleoentorno del asentamiento a la vez que confirma el aprovechamiento de la flora nativa disponible en distintos, pero cercanos nichos ecológicos del área. 
Es significativo en relación al tema del presente trabajo, el cambio que señala la presencia del cultígeno Zea mays en los depósitos del PAT. Si bien la muestra es escasa y los cariopsis presentan fragmentación y deterioro morfológico, debido a la cualidad de sus componentes en almidón al ser sometidos a la acción de la carbonización, algunos son posibles de identificar. Pero el número de restos es notoriamente menor que aquellos correspondientes a Chenopodium quinoa. Estos últimos, aunque exhiben escasa dimensión, no presentan diferencias en su morfología y tamaño al ser comparados con los recuperados en niveles arcaicos. Zea mays fue encontrado también en el Rasgo 1 (fogón del PAT) de la cuadrícula Este, y asociado a la base de un molino en la cuadrícula Oeste (Tablas 4 y 5).

Como Fabaceae, se ha mencionado a restos de legumbres (vainas) y semillas, tales como Vicia sp., Lathyrus sp., frecuentes en la cordillera. En cuanto a Cucurbitaceae, los fragmentos de cáscaras,

Tabla 4. Macrorrestos en el Rasgo 1 (fogón) del PAT entre 44 y $55 \mathrm{~cm}$ de profundidad. Cuadrícula Este.

Late Archaic Period macro remains from Feature 1 (hearth), between 44-55 cm depth, from East Quadrant.

\begin{tabular}{|c|c|c|}
\hline Nivel & $1 \mathrm{c}$ & $1 d$ \\
\hline Litros & 3,5 & 4 \\
\hline Total & 612 & 593 \\
\hline Asteraceae & & $6 *$ \\
\hline Berberis sp. & $2 *$ & \\
\hline Bromus sp. & $65^{*}$ & $47 *$ \\
\hline Caryophyllaceae & $1^{\wedge}$ & \\
\hline Chenopodium album & $12^{\wedge}$ & $6^{\wedge}$ \\
\hline C. quinoa & $123^{*}$ & $34 *$ \\
\hline Chenopodium sp. & $9 *$ & $11 * 10^{\wedge} 1$ desec. \\
\hline Cucurbitaceae & $3 *$ & $4 *$ \\
\hline Cyperaceae & $3 *$ & $1 *$ \\
\hline Discaria chacaye & $10 *$ & $8 *$ \\
\hline Guindilia trinervis & $32 *$ & $1 *$ \\
\hline Juncaceae & & $3 *$ \\
\hline Nicotiana $\mathrm{sp}$. & $10 *$ & $57 *$ \\
\hline Papilionaceae & $2 *$ & \\
\hline Poaceae (ni) & $297^{*} 2^{\wedge}$ & $374 *$ \\
\hline Rosaceae & $1^{*}$ & \\
\hline Scirpus sp. & $13^{*}$ & \\
\hline Zea mays & $1 *(?)$ & $3 *$ \\
\hline No identificados & $26^{*}$ & $26^{*}, 1^{\wedge}$ \\
\hline
\end{tabular}

$*$ = carbonizados

$\wedge=$ no carbonizados

(ni) $=$ no identificada
Tabla 5. Macrorrestos en sedimento asociado a mortero. Cuadrícula Oeste en extensión hacia la boca de alero. Macro remains from soil sediment associated with a mortar. West Quadrant extending toward the opening of the rockshelter.

\begin{tabular}{lc}
\hline Litros & 4 \\
\hline Discaria chacaye & $3^{*}$ \\
Chenopodium quinoa & $11^{*}$ \\
Cyperaceae & $2^{*}$ \\
Cucurbitaceae & $2^{*}(?)$ \\
Oxalix sp. & $1^{\wedge}$ \\
Papilionaceae & $6^{*}$ \\
Poaceae (ni) & $65^{*}$ \\
Zea mays & $4^{*}$ \\
No identificados & $73^{*}$ \\
Total & 167 \\
\end{tabular}

* = carbonizados

$\wedge=$ no carbonizados

(ni) $=$ no identificada

semillas o "pepas" y de pecíolos recuperados denotan, como en los niveles arcaicos, una importante reducción en su tamaño, debida posiblemente a la carbonización y consiguiente deshidratación. No obstante interesa mencionar como antecedente que en un sitio con enterratorios del PAT, a $1.750 \mathrm{msm}$ en el margen sur del curso superior del río Yeso (sitio Chacayes), es decir, en un área relativamente cercana a Las Morrenas 1, se encontraron como ofrendas, réplicas cerámicas de Lagenaria sp. que se asemejan a Lagenaria siceraria (Stehberg 1976). Este dato, aunque indirecto, permite reconocer en los grupos alfareros de este período, la continuación de un interés ya sea económico, funcional u otro por este tipo de vegetales, ya que de hecho las cucurbitáceas han sido representadas no sólo en la alfarería del mencionado sitio, sino también en otros del valle central (Sanhueza 1997).

Las poáceas destacan por la continuidad ininterrumpida en los depósitos desde el Arcaico y sin demostrar cambios en los restos discriminados, en general tres o cuatro, que evidentemente representan un mínimo en relación a las conocidas actualmente para el área. Forman parte de poblaciones vegetales extensas, que pueden alcanzar los hábitats altoandinos hasta los $3.500 \mathrm{msm}$ y que proporcionan una abundante disponibilidad estacional aprovechable para el consumo humano y/o forraje, como algunas del género Bromus destacadas por Matthei (1986) en una recopilación que retrotrae los antecedentes conocidos sobre su utilización, 
hasta los primeros años de la conquista hispana. La significativa cantidad de macrorrestos (cariopsis y tallos) recuperados permite suponer que esta oferta natural de alta disponibilidad no fue indiferente para los ocupantes del alero.

La Tabla 4 representa el contenido de un contexto de fogón o de quema del PAT, en la cuadrícula Este. Tanto la fracción liviana como pesada, luego de la flotación convencional, dan cuenta de una gran cantidad de maderas carbonizadas, y si bien no hay rastros de la presencia de un "piso", se destaca una depositación calcárea que involucra a gran parte de la muestra, particularmente en el nivel 1d. El hallazgo, en la fracción pesada, de una cuenta de collar de malaquita de $5 \mathrm{~mm}$ de diámetro, entre restos de talla lítica y osteofauna abundantes, además de los tipos cerámicos, vincula a este grupo social con los contemporáneos del valle central, en cuyos contextos habitacionales y principalmente de funebria es frecuente encontrar este tipo de adorno.

De este rasgo de fogón o quema, con 7,5 litros flotados, se obtuvieron 1.205 macrorrestos, de los cuales sólo 33 no estaban carbonizados. Los resultados del análisis efectuado en este nivel entre 44 y $52 \mathrm{~cm}$ de profundidad, refuerzan la información sobre la variabilidad de especies existente alrededor del año 400 d.C. en el ecosistema altoandino. Los restos vegetales son similares a aquellos provenientes de los depósitos culturales de los mismos niveles en las columnas de muestreo, probablemente debido a la dispersión de los restos y la proximidad de dicho rasgo con el sector donde se practicó una de las columnas. El cambio más significativo lo constituyen las cantidades de especímenes recuperados, lo que efectivamente puede responder a las características funcionales de un fogón, con gran abundancia de maderas y Poaceae carbonizadas en muy buen estado de conservación.

En este contexto, Chenopodium quinoa aparece representada por 157 semillas $(13,02 \%)$, destacándose cuatro de ellas por un gran parecido a "cañihua" o Chenopodium pallidicaule, y tal como se aprecia en la columna de muestreo, con una apreciable mayor cantidad de restos que los de Zea mays.

Del mismo modo que en la columna de muestreo de la cuadrícula Este, destaca entre los depósitos del mencionado fogón una cantidad considerable de semillas carbonizadas de Nicotiana sp. Esta situación es interesante, ya que en sitios del PAT en el valle central se han efectuado hallazgos de numerosas pipas para fumar, pero no han sido encontradas estas semillas en los estudios arqueobotánicos correspondientes (Planella y Tagle 1998).

En la cuadrícula Oeste, entre los 55 y $60 \mathrm{~cm}$ de profundidad en la boca del alero rocoso, se extrajo cuatro litros de tierra asociada a la base de un molino confeccionado sobre un bloque de andesita. La muestra (Tabla 5) es muy generosa en distintas Poaceae carbonizadas, cuya presencia puede responder a forraje puesto a resguardo, a manera de silo, aprovechando la protección del alero; pero es interesante notar que todas ellas están carbonizadas, lo que más bien puede indicar que sus granos tostados eventualmente estaban siendo objetos de un procesamiento o molienda en dicho instrumento; sin descartar que también hayan sido utilizadas como pastos secos para encender fuegos.

En este sector, además de un cariopsis de Zea mays con sus rasgos morfológicos en buenas condiciones, se logró identificar una cúpula. La representatividad de este cultígeno en las muestras es escasa, pero igualmente significativa al encontrarse estas evidencias asociadas al instrumento de molienda mencionado, y al vincular estos depósitos con otros del valle central en que se ha constatado la presencia de este alimento en el transcurso del PAT. Las vainas y semillas de Fabaceae recuperadas en este contexto son muy pequeñas, y parecen corresponder a las especies que ya se han señalado.

En cuanto a Chenopodium quinoa, se obtuvieron 11 ejemplares, algunos partidos, pero con sus contornos muy bien conservados. Su asociación espacial con respecto a la ubicación del molino podría indicar que tanto este recurso alimenticio de alto valor proteico, algunas Poaceae, entre ellas el maíz, pudieron ser procesados para la obtención de harinas y alimentación de los ocupantes del alero.

Por debajo del contexto anterior, que incluye microlascas y desechos líticos además de fragmentos óseos de guanaco, aves y roedores, en las excavaciones en la boca del alero se encontraron tres manos de moler a una profundidad de 110 a 120 $\mathrm{cm}$. Una de ellas presentaba huellas de pigmento rojo en la superficie y otra, de forma circular con rebaje en una cara y en el canto, son características del Arcaico regional. La muestra de tierra tomada de este sector es bastante escasa, sólo 0,25 litros, y proporcionó regular cantidad de madera carbonizada, junto con una falange humana y un ejemplar de Chenopodium quinoa.

Los resultados obtenidos en los análisis arqueobotánicos, como se ha podido apreciar, fueron po- 
sitivos en cuanto a la existencia de cultígenos, destacando la presencia de Chenopodium quinoa desde los niveles más antiguos del asentamiento estudiado. Con el objetivo de esclarecer la variable cronológica y definir con mayor certeza la confiabilidad de los resultados, se envió a datación radiocarbónica por AMS una muestra de 24 ejemplares carbonizados de Chenopodium quinoa tomados de los niveles inferiores en las columnas de muestreo de las cuadrículas Este $(\mathrm{n}=12)$ y Oeste $(\mathrm{n}=12)$, entre 90 y $110 \mathrm{~cm}$ de profundidad. Ello proporcionó una fecha radiocarbónica convencional de $2960 \pm 40$ a.p., o de 3.250-2.980 años cal. a.p. (BETA 146230) con una probabilidad de $95 \%$, y que concuerda con el rango de fechas obtenidas para estos niveles arcaicos a partir de las muestras de carbón mencionadas anteriormente. De este modo se corrobora la temprana presencia de este cultígeno entre cazadores recolectores de fines del Arcaico.

Por otra parte, el estudio arqueobotánico apoya indiscutiblemente el carácter estacional de las ocupaciones humanas en Las Morrenas 1, condicionadas probablemente porque el alero se encuentra sobre los $2.400 \mathrm{~m}$ de altitud por encima del límite de las nieves de invierno, lo que haría disponible este asentamiento sólo desde mediados de la primavera hasta mediados de otoño. Esta condición probablemente existió también hace 4.000 años.
En cuanto a entender la finalidad de uso del alero Las Morrenas 1, el tipo de instalación descrito aquí como campamento de tareas para ambas ocupaciones, está definido básicamente por la extremada especialización tecnológica, que es evidente al estudiar las funciones de los instrumentos líticos provenientes de los contextos arqueológicos (Tabla 6). Si bien el contexto del PAT es más diverso que el del Arcaico IV (PAT $H=0,80$ Arcaico IV $H=0,59^{4}$ ), es evidente que los instrumentos descartados en el sitio se concentran en ciertas funcionalidades específicas. Los instrumentos más frecuentes son los cuchillos y los raspadores, los cuales en ambas ocupaciones representan cerca del $70 \%$ de los instrumentos. Los instrumentos como categoría, por lo demás, se encuentran en una densidad muy alta, al menos comparada con la representación que tienen en otros asentamientos de la región, alcanzando en conjunto más de 11 ejemplares por litro (Cornejo et al. 2000a; Peralta y Salas 2000, 2004). La mayor parte de estas herramientas fue confeccionada con sílice opaco, parte del cual, de acuerdo a una prospección realizada por Sebastián Ibacache comunicación personal (2003), podría provenir del portezuelo Peladeros, a unos $8 \mathrm{~km}$ de distancia y a $3.300 \mathrm{~m}$ de altitud.

Lo anterior remite al tipo de actividades que se realizaron en el sitio, las cuales en una parte importante estarían relacionadas precisamente con

Tabla 6. Instrumentos por períodos en el sitio Las Morrenas 1.

Lithic tools by period at the Las Morrenas 1 rockshelter site.

\begin{tabular}{|c|c|c|c|c|c|c|}
\hline \multirow[t]{2}{*}{ Período } & \multicolumn{2}{|c|}{ Arcaico IV } & \multirow[b]{2}{*}{ Densidad* } & \multicolumn{3}{|c|}{ Alfarero Temprano } \\
\hline & $\mathrm{N}$ & $\%$ & & $\mathrm{~N}$ & $\%$ & Densidad* \\
\hline Puntas de Proyectil & 6 & 10,0 & 1,5 & 4 & 6,5 & 1,5 \\
\hline Puntas o Cuchillos & 8 & 13,3 & 2,1 & 5 & 8,1 & 1,8 \\
\hline Cepillos & 1 & 1,7 & 0,3 & 1 & 1,6 & 0,4 \\
\hline Cuchillos Bifaciales & 5 & 8,3 & 1,3 & 4 & 6,5 & 1,5 \\
\hline Cuchillos No Bifaciales & 11 & 18,3 & 2,8 & 11 & 17,7 & 4,0 \\
\hline Cuchillos N/B o Raederas & & 0,0 & & 1 & 1,6 & 0,4 \\
\hline Microrraederas & 1 & 1,7 & 0,3 & 1 & 1,6 & 0,4 \\
\hline Perforadores & & 0,0 & & 3 & 4,8 & 1,1 \\
\hline Raspadores & 24 & 40,0 & 6,2 & 27 & 43,5 & 9,8 \\
\hline Raspadores o Cuchillos & 2 & 3,3 & 0,5 & 2 & 3,2 & 0,7 \\
\hline Raederas & & 0,0 & & 1 & 1,6 & 0,4 \\
\hline Manos de Moler & 2 & 3,3 & 0,3 & 2 & 3,2 & 0,7 \\
\hline Total general & 60 & & & 62 & & \\
\hline
\end{tabular}

* Instrumentos por $\mathrm{m}^{3}$. 



carece de otros antecedentes, más allá del tipo de herramientas de piedra, es posible proponer que las tareas específicas desarrolladas en el sitio, tanto durante el Arcaico IV como en el PAT, tuvieron que ver con la preparación de pieles de animales. Este es el único recurso potencial de la localidad que podría justificar la extremada especialización funcional descrita. Pese a que pequeñas ramas carbonizadas observadas bajo lupa presentan huellas de cortes muy nítidas, a la altitud en que se encuentra el sitio no hay vegetación arbórea para ser procesada con los instrumentos que caracterizan este contexto; mientras que la notable abundancia de pastos y la inusual concentración de recursos hídricos en la localidad (varias lagunas, esteros y vegas) la hacen muy adecuada para la presencia de animales que podrían proveer el recurso pieles, además de carne.

\section{Discusión}

En este escenario natural y cultural de Chile Central, los datos y resultados del estudio interdisciplinario realizado, confirman la presencia de cultígenos en contextos de cazadores recolectores de finales del período Arcaico, hace aproximadamente 3.000 años. Se discuten a continuación las implicancias de estos hallazgos para la arqueología de la región.

En América del Sur los indicadores más antiguos de manipulación de recursos vegetales provienen de sitios de la cordillera andina. Pese a que es necesario considerar que existen problemas de sesgos en algunos datos, debido a estratigrafías confusas o a intrusiones en los contextos y carencias de orden cronológico, es posible admitir, de acuerdo a numerosos estudios, que existen elementos que validan la presencia de precoces prácticas de manipulación de ciertas especies vegetales a partir de aproximadamente 5.000 años a.C., en lo que concierne a países como Argentina, Bolivia, Chile y Perú (Lavallèe 2000).

En la actualidad ha habido un significativo aumento de los datos, que documenta más sobre los procesos naturales y culturales involucrados en el problema de la emergencia de los primeros cultígenos (Castro y Tarragó 1992; Núñez 1989). Algunos trabajos han sido relativamente concluyentes en relación a ciertos recursos, pero persis- ten interrogantes de gran interés con respecto a otros, como es el caso de Chenopodium quinoa discutido por diversos investigadores (Gremillion 1993; Pearsall 1989; Pickersgill 1989; Rossen et al. 1996; Wilson 1988), ya sea considerando problemas de filogenia, de caracteres morfológicos diagnósticos de domesticación, o de la larga persistencia en el tiempo de ciertos rasgos como el pequeño tamaño de los especímenes antes de evidenciar cambios en tal sentido.

La presencia de este seudocereal (Chenopodium quinoa), en niveles con depósitos de cazadores recolectores en la cordillera de Chile Central, abre una nueva perspectiva para las investigaciones que se están realizando en el cono sur de América relacionadas con dichos grupos. El estudio arqueobotánico en Las Morrenas 1 ha permitido constatar que las características morfológicas de los especímenes carbonizados analizados son las mismas que presentan aquellos provenientes de sitios arqueológicos del PAT de la región central de Chile (Planella y Tagle 1998, 2004), características que también son compartidas por especímenes carbonizados de sitios como la Cueva de Panaulauca, en Perú (Pearsall 1989; Pearsall comunicación personal 2000), y sitios en Catamarca, Argentina (Rossen comunicación personal 1999; Rodríguez 2004). De este modo es posible extender los datos sobre cultígenos iniciales a estas latitudes.

El hallazgo de cultígenos en el ámbito que se ha detallado, no constituye una novedad cronológica en el concierto de datos de contextos arcaicos que han sido publicados en otras áreas de Sudamérica y en la región trasandina (Gil 1997-98), pero sí se debe destacar su relevancia para la arqueología de la región central de Chile, ya que constituye una información nueva y trascendental que modifica sustancialmente la tradicional apreciación cualitativa de las sociedades de fines del Arcaico en esta área. Queda demostrado un cambio que afecta sus mecanismos de subsistencia, que aporta otras proteínas y mayor balance a la dieta, y un paso crucial para procurar en adelante el surgimiento de las prácticas hortícolas que se confirman en el PAT.

Si bien no se logra explicar cómo y en qué circunstancias los restos de Chenopodium quinoa llegaron a integrar los depósitos dejados desde los inicios de la ocupación del alero, es posible plantear que su presencia se debe a la movilidad que acusan estos grupos, con los consiguientes benefi- 
cios selectivos del contacto con otros con experiencias previas en manipulación de Chenopodiaceae. El circuito de movilidad logística propuesto para estos grupos seguramente se vincula con un espacio muy amplio, ya que la posición del sitio, muy cerca del paso Piuquenes, que comunica las dos vertientes de la cordillera de los Andes, permite tanto el acceso hacia los valles de Chile Central como a la actual provincia argentina de Cuyo. De hecho, la movilidad hacia la vertiente oriental de los Andes parece ser una de las posibles respuestas a la presencia de quinoa en el contexto Arcaico IV de Las Morrenas $1^{6}$. Este tipo de evidencias no ha sido detectada en sitios, localizados en la parte más baja de la cuenca cordillerana del río Maipo que son contemporáneos a esta ocupación (Cornejo et al. 2000a, 2000b; Peralta y Salas 2000, 2004).

La presencia de restos óseos de guanaco (Lama guanicoe) desde el registro Arcaico, sugiere también algunos aspectos determinantes de interacción de los grupos humanos que ocasionalmente habitaban el alero con el recurso faunístico silvestre representado por esta especie, abundante, al parecer, en el área, y a la vez directa e indirectamente con la flora nativa del macizo andino. Como cazadores, el seguimiento de estos animales por rutas ya conocidas y otras alternativas, les permitió acceder a entornos que probablemente estimularon nuevas experiencias de recolección vegetal. En el caso de las poáceas, se sugiere que estas constituían parte de la alimentación de los guanacos, debido a la amplia cobertura espacial de su crecimiento espontáneo y su alto índice de germinación estacional en el ámbito andino. La recolección de sus granos maduros por parte de integrantes de dichos grupos, tal como se ha descrito para otras culturas, debe haber sido eficiente aunque siempre parcial, ya que la abundancia y caducidad de los granos al alcanzar su madurez hacen difícil su recolección total (Willcox 2000). A su vez, este proceso natural de las poáceas da como resultado una disponibilidad asegurada para la siguiente temporada de germinación en los mismos sitios, sin necesidad de realizar los individuos tareas especiales para lograr su obtención (Anderson 2000).

Si bien lo anterior fue, sin duda, de gran importancia para los cazadores recolectores, la manipulación de algunas especies vegetales con mayor valor nutricional, como lo es la quinoa con su alto contenido en proteínas, no se comprende sino con el previo conocimiento paulatinamente adquirido directa o indirectamente en relación a sus potenciales de abundancia y posibilidades de almacenaje. Esta comprensión cualitativa de ciertos recursos vegetales debe haber estimulado la preocupación de los grupos hacia la búsqueda de condiciones de sustento más diversificadas, generando conceptos y materialidades adecuadas para resguardar los alimentos, con el fin de asegurar y prolongar su disponibilidad a través del ciclo anual, sentando de este modo las bases que habrían contribuido para diferir la movilidad, alternando con períodos de mayor permanencia en los sitios.

Pero, ¿tenían estos cazadores-recolectores elementos materiales apropiados para garantizar un adecuado almacenaje? En las excavaciones realizadas en Las Morrenas 1 no se detectó ningún rasgo, dentro del contexto espacial del alero rocoso y su entorno inmediato, que permita sugerir empíricamente la existencia de un locus especialmente acondicionado para cumplir con esta función. Por otra parte, si bien en el registro arqueobotánico correspondiente a los niveles arcaicos se detectaron restos de semillas y cáscaras de Cucurbitaceae, no fue posible comprobar que especímenes de Lagenaria sp. fuesen utilizados con dicho propósito, tal como sí ha ocurrido en otros sitios arcaicos del Área Andina (Gambier 1977). Pese a las carencias mencionadas, es importante tener presente que los granos (frutos) de quinoa sin procesar, es decir, sin ser sometidos a suficientes lavados, están recubiertos por un pericarpio con alto contenido en saponinas, lo que les confiere un sabor muy amargo que no los hace apetecibles incluso para los roedores, $\mathrm{y}$, por tanto, es posible que fuesen almacenados sin requerir de una protección especial. Esta característica pudo haber constituido un ventajoso factor de estímulo para la adopción temprana de este alimento rico en proteínas, antes que el maíz (Zea mays).

La presencia de Chenopodium quinoa de tamaño pequeño, en un contexto de cazadores recolectores del Arcaico IV, no responde necesariamente a una situación resultante de un proceso local de manejo de una especie silvestre de Chenopodiaceae; la existencia de ésta integrando la cubierta vegetal regional, en las fases más temprana del Arcaico, es un hecho comprobado por estudios de polen (Heusser 1983; Rojas 1991) y por recientes investigaciones arqueobotánicas en cuencas paleolacustres de la zona central de Chile (Planella y Tagle 2004; Tagle y Del Río 2000). Sin embargo, en los estudios hasta ahora realiza- 
dos en el valle central no se verifican signos morfológicos de manipulación antrópica de estas plantas hasta el PAT (Belmar y Quiroz 2000; Planella y Tagle 1998, 2004).

Si bien la información que provee el sitio cordillerano de Las Morrenas 1 corresponde por el momento a una excepción dentro del Arcaico regional, no lo es en relación a las ya mencionadas áreas vecinas, donde se han localizado sitios, también en ámbitos de la cordillera de los Andes, que pudieron haber constituido tempranos umbrales proveedores de cultígenos durante procesos de contactos e intercambio cultural. Por otra parte, no se puede establecer que el factor altitudinal afecte negativamente la presencia de Chenopodiaceae en la franja cordillerana, ya que en sectores resguardados existen las condiciones naturales para el desarrollo de estas especies. Ello permite considerar como eventual que los cazadores recolectores del área en estudio, ya al final del Arcaico, hayan procurado habilitar espacios reducidos en quebradas u otros para ensayar y adoptar algunos cultígenos (Dillehay et al. 1989). No obstante, las características climáticas a más de $2.000 \mathrm{msm}$ con nieves y heladas prolongadas, hacen poco probable el cultivo de Chenopodiaceae en el entorno de Las Morrenas 1. En esas condiciones los frutos de Chenopodium quinoa requerirían más de cinco meses para lograr su madurez, lo que sería crítico, de acuerdo a los plazos de cosecha que permiten las nieves. El modo de vida cazador-recolector de alta movilidad que marcó el período Arcaico IV se mantuvo vigente no sólo durante las ocupaciones del PAT, sino que hasta tiempos tardíos, al menos en la región cordillerana (Cornejo y Sanhueza 2003; Cornejo et al. 1998; Saavedra y Cornejo 1995). En el transcurso de las primeras fases de ese modo de vida, se inicia la incorporación de algunos recursos vegetales cultivados que han sido sustanciales en el desarrollo de las poblaciones del Área Andina.

Los resultados alcanzados en esta investigación interdisciplinaria son, sin duda, relevantes, pero la complejidad de los temas tratados necesariamente exige continuar con este tipo de estudios en Chile Central, particularmente en otros sitios de la cordillera.

Agradecimientos: El estudio del sitio Las Morrenas 1 se realizó en el marco del proyecto FONDECYT 1970071.

\section{Referencias Citadas}

Anderson, P.

2000 La Tracéologie comme révélateur des débuts de l'agriculture. En Premiers Paysans du Monde, pp. 99-119. Editions Errance, Paris.

Binford, L.

1980 Willow smoke and dogs'tails: Hunter-gatherer settlement system and archaeological site formation. American Antiquity 45:4-20.

Belmar, C. y L. Quiroz

2000 Informe arqueobotánico: sitio El Cebollar. Informe Proyecto Fondecyt N $\mathrm{N}^{\circ}$ 1900667. Manuscrito en posesión de los autores.

Castro, V. y M. Tarragó

1992 Los inicios de la producción de alimentos en el cono sur de América. Revista de Arqueología Americana 6:91-124.

Cornejo, L. y L. Sanhueza

2003 Coexistencia de cazadores recolectores y horticultores tempranos en la cordillera andina de Chile Central. Latin American Antiquity 14:389-407.

Cornejo, L. y J. Simonetti

1992 Asentamientos prehistóricos en los Andes de Chile Central: Tradición y flexibilidad. Clava 5:81-98.

1993 Asentamiento humano en los Andes de Chile Central: Un enfoque alternativo. Actas del XII Congreso de Arqueología Chilena, Tomo II:373-380. Sociedad Chilena de Arqueología. Temuco.
1997-98 De rocas y caminos: espacio y cultura en los Andes de Chile Central. Revista Chilena de Antropología 14: 127-143.

Cornejo, L., M. Saavedra y H. Vera

1998 Periodificación del Arcaico en Chile Central: Una propuesta. Boletín de la Sociedad Chilena de Antropología 25:36-39.

2000a Asentamientos arcaicos tardíos en El Manzano (Río Maipo). Actas del XIV Congreso Nacional de Arqueología Chilena. Contribución Arqueológica $\mathrm{N}^{\circ}$ 5, Tomo I:621-636. Sociedad Chilena de Arqueología, Copiapó.

2000b Informe final Proyecto Fondecyt 1970071. http:// www.arqueologia.cl/andes/informe.htm

Dillehay, T.D., P.J. Netherly y J. Rossen

1989 Middle preceramic public and residential sites on the forested slope of the western Andes, northern Peru. American Antiquity 54:733-759.

Evans, J. y T. O’Connor

2001 Environmental Archaeology. Principles and Methods. Sutton Publishing, Stroud.

Gajardo, R.

1994 La Vegetación Natural de Chile. Clasificación y Distribución Geográfica. Editorial Universitaria, Santiago.

Galarce, P. y P. Peralta

2003 Organización tecnológica lítica y movilidad de grupos cazadores recolectores en asentamientos cordilleranos del 
Arcaico tardío en Chile Central. Ponencia presentada en el XVI Congreso Nacional de Arqueología Chilena, Tomé.

Gambier, M.

1977 La cultura de Ansilta. Instituto de Investigaciones Arqueológicas y Museo, Universidad de San Juan, San Juan.

Gil, A.F.

1997-98 Cultígenos prehispánicos en el sur de Mendoza. Discusión en torno al límite meridional de la agricultura andina. Relaciones de la Sociedad Argentina de Antropología XXII-XXIII:295-318.

Gremillion, K.J.

1993 The evolution of seed morphology in domesticated Chenopodium: an archaeological case study. Journal of Ethnobiology 13:149-169.

Heusser, C.

1983 Quaternary pollen record from Laguna de Tagua-Tagua, Chile. Science 219:1429-1432.

Hoffmann, A., M. Kalin, F. Liberona, M. Muñoz y J. Watson 1998 Plantas Altoandinas en la Flora Silvestre de Chile. Ediciones Fundación Claudio Gay, Santiago.

Lavallèe, D.

2000 Les premiers producteurs de l'Amerique du Sud. En Premiers Paysans du Monde, pp. 191-211. Editions Errance, Paris.

Matthei, $\mathrm{O}$.

1986 El Género Bromus L.(Poaceae) en Chile. Gayana Botánica 43:47-110.

Núñez, L.

1989 Hacia la producción de alimentos y la vida sedentaria (5.000 a.C. a 500 d.C.) En Prehistoria, desde sus Orígenes hasta los Albores de la Conquista, editado por J. Hidalgo, V. Schiappacasse, H. Niemeyer, C. Aldunate e I. Solimano, pp. 81-105. Editorial Andrés Bello, Santiago.

Peralta, P. y C. Salas

2000 Patrones de asentamiento de cazadores-recolectores cordilleranos: Una categoría particular de sitio arqueológico. Boletín de la Sociedad Chilena de Arqueología 29:20-30.

2004 Funcionalidad de asentamientos cordilleranos durante el Arcaico tardío y el Agroalfarero temprano (Chile Central). Chungara Revista de Antropología Chilena, Volumen Especial, Tomo II:923-933.

Pearsall, D.M.

1989 Adaptation of prehistoric hunter-gatherers to the high Andes: the changing role of plant resources. En Foraging and Farming. The Evolution of Plant Explotation, editado por D.R. Harris y G.C. Hillman, pp. 318-332. Institute of Archaeology, University College London.

Pickersgill, B.

1989 Cytological and genetical evidence on the domestication and diffusion of crops within the Americas. En Fora- ging and Farming. The Evolution of Plant Explotation, editado por D.R. Harris y G.C. Hillman, pp. 426-439. Institute of Archaeology, University College London.

Planella, M.T. y B. Tagle

1998 El sitio Agroalfarero de La Granja: un aporte desde la perspectiva arqueobotánica. Publicación Ocasional $\mathrm{N}^{\circ}$ 52. Museo Nacional de Historia Natural de Chile, DIBAM, Santiago.

2004 Inicios de presencia de cultígenos en la Zona Central de Chile, períodos Arcaico y Agroalfarero Temprano. Chungara Revista de Antropología Chilena, Volumen Especial, Tomo II:387-400.

Rodríguez, M.F.

2004 Cambios en el uso de los recursos vegetales durante el Holoceno en la Puna Meridional Argentina. Chungara Revista de Antropología Chilena, Volumen Especial, Tomo II:403-413.

Rojas, G.

1991 Posibilidades de alimentación vegetal del Hombre de Cuchipuy. Revista Chilena de Antropología 10:25-35.

Rossen, J., T. Dillehay y D. Ugent

1996 Ancient cultigens or modern intrusions?: evaluating plant remains in an Andean case study. Journal of Archaeological Science 23:391-407.

Sanhueza, L.

1997 Relaciones llano-precordillera Durante el Período Agroalfarero Temprano en Chile Central: Una visión Desde la Cerámica. Memoria para optar al Título de Arqueóloga, Departamento de Antropología, Facultad de Ciencias Sociales, Universidad de Chile, Santiago.

Saavedra, M. y L. Cornejo

1995 Acerca de la cronología de El Manzano. Boletín de la Sociedad Chilena de Arqueología 21:31-34.

Stehberg, R.

1976 El cementerio Alfarero Temprano de Chacales. Interior del Cajón del Maipo (Datado en 430 d.C.). Actas y Memorias Cuarto Congreso Nacional de Arqueología Argentina. Tomo III:277-295, Mendoza.

Tagle, B. y C. del Río

2000 Túmulos funerarios en cuencas palustres del valle del río Tinguiririca. Manuscrito en posesión de las autoras.

Tagle, B. y M.T. Planella

2002 La Quinoa en la Zona Central de Chile. Supervivencia de una Tradición Prehispana. Editorial IKU, Santiago.

Willcox, G.

2000 Nouvelles données sur l'origine de la domestication des plantes au proche Orient. En Premiers Paysans $d u$ Monde, pp. 123-139. Editions Errance, Paris.

Willson, H.D.

1988 Quinua biosystematics II: free-living populations. Economic Botany 42:478-494.

\section{Notas}

1 No se efectuó la identificación de los residuos de maderas, pero se cuantificó estimativamente su abundancia o escasez en los diferentes rasgos registrados, para una posterior interpretación.

2 En los sitios arqueológicos de Chile Central los granos de Chenopodium quinoa se encuentran carbonizados. Esta con- dición, además de la pérdida de agua y otros componentes que se volatilizan, no influye tanto en la morfología, sino que en el tamaño, el cual es alrededor de un $30 \%$ menos que en la quinoa no carbonizada. Esto ha llevado a algunos investigadores a preferir referirse a ellos como "tipo quinoa". Una descripción más amplia se encuentra en Planella y Tagle (1998). 
3 Una de estas especies de Poaceae fue identificada como Bromus setifolius J.S. Presl, por el Profesor Luis Faúndez, taxónomo de la Universidad de Chile. Esta planta es conocida como recurso forrajero.

$4 \mathrm{H}=$ Índice de Diversidad de Shannon expresado con relación a la diversidad máxima posible que adquiere el valor $H=1,0$.

5 Dado que muchas de las herramientas presentan evidencias de haber sido utilizadas y que las materias primas con que se confeccionaron no son locales, se ha descartado la posibilidad de que la abundancia de este tipo de herramien- tas se deba a que este asentamiento esté relacionado específicamente con su producción.

6 El análisis bajo lupa binocular de muestras de granos de quinoa de la Gruta del Indio (cordillera de Cuyo), facilitadas por Adolfo Gil, muestra una clara similitud morfológica y de tamaño con aquellos de las ocupaciones Arcaicas y del PAT de Las Morrenas 1, aun cuando los primeros se encuentran desecados y los últimos carbonizados. A su vez, las dos muestras se distancian en el tamaño de los granos, comparados con los de Ansilta (San Juan) que fueron facilitados por C. Teresa Michieli, que también están desecados, pero son ligeramente más grandes. 